\title{
Belgeo
}

\section{"To be in the place ": les open mics comme espaces de légitimation artistique pour les scènes rap à Paris et Atlanta}

"To be in the place": open mic events as spaces of legitimacy in the rap scenes of Paris and Atlanta

\section{Séverin Guillard}

\section{(2) OpenEdition}

\section{Journals}

Édition électronique

URL : http://journals.openedition.org/belgeo/13025

DOI : 10.4000/belgeo.13025

ISSN : 2294-9135

Éditeur :

National Committee of Geography of Belgium, Société Royale Belge de Géographie

\section{Référence électronique}

Séverin Guillard, « «To be in the place » : les open mics comme espaces de légitimation artistique pour les scènes rap à Paris et Atlanta », Belgeo [En ligne], 3 | 2014, mis en ligne le 19 décembre 2014, consulté le 03 mai 2019. URL : http://journals.openedition.org/belgeo/13025 ; DOI : 10.4000/ belgeo.13025

Ce document a été généré automatiquement le 3 mai 2019.

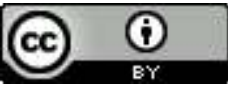

Belgeo est mis à disposition selon les termes de la licence Creative Commons Attribution 4.0 International. 


\title{
"To be in the place ": les open mics comme espaces de légitimation artistique pour les scènes rap à Paris et Atlanta
}

\author{
"To be in the place": open mic events as spaces of legitimacy in the rap scenes of \\ Paris and Atlanta
}

Séverin Guillard

1 Cet article se propose d'observer le fonctionnement d'une scène locale en étudiant les pratiques spatiales présentes dans des concerts dédiés à la musique rap en France et aux Etats-Unis.

2 Si les phénomènes artistiques n'ont suscité un intérêt conséquent que récemment dans la géographie francophone, le rôle de l'espace dans le rap est reconnu depuis bien longtemps par les chercheurs. Cette musique a souvent été décrite comme prenant sa source dans les conditions de vie urbaines des artistes (Forman, 2002 ; Rose, 1994). Cependant, beaucoup d'études se sont contentées d'examiner ce lien à l'espace à travers les discours présents dans les morceaux ou dans les clips, considérant que ceux-ci reflétaient de manière « naturelle » la vie menée par les rappeurs. Or, loin de se limiter à un ensemble d'œuvres caractérisées par un format spécifique, le rap correspond aussi à des mondes sociaux investis par des individus liés à ce genre musical. C'est au sein d'espaces dédiés à la production artistique que vont s'élaborer les codes, les discours et les styles qui se retrouvent ensuite dans la musique.

3 Cette analyse se concentrera sur deux régions urbaines : celles de Paris et d'Atlanta. L'objectif d'une telle comparaison est de relativiser l'approche généralement employée en sciences sociales pour observer la diffusion du rap. Cette musique y est souvent considérée sous l'angle d'une culture "globale » (Mitchell, 2001), qui viendrait s'adapter à des "particularités locales» (Rérat, 2006), selon un processus bien identifié de "glocalisation ». Or, de ce fait, les analyses tendent à fixer une identité musicale stable et 
uniforme sur un territoire spécifique, comme si les styles musicaux étaient la conséquence naturelle de caractéristiques existant en tant que telles sur des espaces locaux.

4 A l'instar de réflexions récentes en géographie de la musique, cet article a ainsi pour objectif de contribuer à « 'désencarter' une géographie construite sur des représentations illusoires » (Raibaud, 2011). Pour cela, le cadre de l'analyse sera déplacé vers un angle quelque peu différent de celui adopté habituellement : celui des scènes artistiques locales. On s'inspirera pour cela de l'approche par les scènes, développée au cours des dernières décennies dans le champ des Popular Music Studies. Will Straw introduit la notion de «scène locale » dans un article de 1991 pour désigner un « espace culturel dans lequel une diversité de pratiques coexiste $»^{1}$. Son objectif est alors d'appréhender la mondialisation de la musique sous l'angle de l'hétérogénéité des pratiques qui en résultent dans les grands centres urbains. Largement reprise et enrichie depuis, la "perspective de la scène» (Bennett, 2004) met l'accent sur les espaces dans lesquels se déploie la pratique musicale en considérant celle-ci sous ses différentes dimensions, de la production à la diffusion et à la réception.

6 Ces réflexions autour des scènes présentent ici deux grands intérêts. Elles invitent d'une part à passer d'une géographie des représentations, fondée sur l'analyse d'un matériau musical, à une géographie des pratiques artistiques. La scène permet en effet de se pencher sur les contextes de production du matériau musical, en observant la manière dont des "groupes de producteurs, musiciens et fans partagent collectivement leurs goûts musicaux et se distinguent des autres ${ }^{2} »$ (Bennett, Peterson, 2004, p. 1).

7 D'autre part, les chercheurs insistent sur l'instabilité des relations qui se tissent entre les individus au sein des scènes. Les caractéristiques des musiques provenant d'un lieu sont considérées comme un compromis provisoire, changeant en fonction de « la création et la reconfiguration des alliances ${ }^{3}$ " (Straw, 1991) entre les individus et de la place de la scène dans le monde musical à une échelle plus large. Plutôt que d'analyser la musique produite comme quelque chose de donné, il s'agit ainsi de l'observer comme le résultat de normes élaborées dans des contextes artistiques spécifiques. Entrer par l'angle de la scène permet donc d'interroger les dynamiques de production du local.

On se concentrera ici sur les contextes de production des œuvres de rap en abordant les pratiques spatiales que l'on retrouve dans des événements clés pour ce genre musical : les open mics. Se focaliser sur ces manifestations de type "concert» présente en effet l'intérêt de mettre l'accent sur des pratiques en train de se faire plutôt que sur les représentations déjà fixées que véhiculent les produits musicaux. Par ailleurs, de par leur statut de porte d'entrée dans le monde du rap, les open mics constituent un terrain d'observation particulièrement riche pour observer la manière dont se structure l'espace spécifique des scènes locales.

9 Après avoir problématisé les open mics comme objet d'étude pour analyser les scènes du rap à Paris et à Atlanta, on observera les pratiques et les discours se déroulant dans les événements des deux villes. On montrera par là comment se construisent deux modes de légitimation différenciés, propres à chacune des scènes. On analysera ainsi comment ces pratiques révèlent deux modèles de capitales du rap, qui nous renseignent sur la manière dont se hiérarchisent et se structurent les mondes du rap en France et aux Etats-Unis. 


\section{Les scènes rap au prisme des open mics}

10 Cet article vise à comprendre l'ancrage spatial du rap comme celui d'une scène artistique à part entière. Les open mics constituent pour cela un bon terrain d'observation : ils sont en effet le lieu de pratiques qui reflètent les normes de la scène et participent à leur évolution.

\section{Les open mics : définition et intérêt de l'objet d'étude}

11 Dans le domaine du rap, le terme d'open mic désigne un événement s'apparentant à une scène ouverte. Le micro y est disponible pour toute personne désirant interpréter quelques morceaux ou quelques couplets, moyennant généralement l'inscription préalable sur une liste ou le paiement d'un droit quelconque.

Dans les deux villes, cette étiquette est utilisée par les acteurs pour qualifier des événements de type assez divers. En effet, ce n'est pas tant un format uniforme qui semble définir les open mics que quelques grands traits caractéristiques qui les distinguent d'autres types de concerts dédiés au genre rap. D'une part, les modes de sélection et de rétribution des artistes les opposent à des spectacles organisés sur la base d'une direction artistique: la présence sur scène des artistes dépend non pas du choix d'un programmateur mais d'une inscription ou d'un paiement de la part des rappeurs euxmêmes, rendant ainsi ces événements accessibles à des individus situés hors des réseaux professionnels et dominants du rap. Cette différence dans le mode de sélection se traduit cependant aussi par une absence de rémunération des artistes se produisant sur scène. D'autre part, les open mics se démarquent également des locations de salles, parfois effectuées par certains artistes afin d'assurer leur propre promotion : on y trouve en effet une distinction stricte entre les individus qui organisent l'événement et ceux qui s'y produisent. Enfin, c'est le grand nombre d'artistes présents sur scène qui caractérise les open mics, les distinguant en cela de toute forme de concert orienté autour d'un ou de quelques groupes.

Les open mics constituent ainsi un terrain particulièrement riche pour montrer les logiques selon lesquelles s'organise une scène dans une localité et une temporalité donnée. En effet, de par leur accessibilité, ce type de dispositif constitue pour les artistes une des portes d'entrée dans la scène rap, avant même la signature sur un label ou le passage en radio. Dans leurs pratiques, les artistes vont donc se placer par rapport à ce qu'ils imaginent être les normes de la scène au moment où ils désirent s'y intégrer.

Par ailleurs, les open mics en eux-mêmes possèdent une certaine place au sein de la scène. De par les artistes qui s'y produisent, ils tendent à regrouper la frange dite « underground ${ }^{4} »$ de celle-ci. Pourtant, la notoriété de certains événements s'étend parfois bien au-delà de la scène locale. Il arrive ainsi d'entendre, à Atlanta, des rappeurs déclarer venir de villes comme Saint Louis, Miami ou New York et avoir fait la route spécialement pour se produire. Ainsi, l'étude des open mics permet d'analyser le rôle joué par cette frange de la scène dans la définition et l'évolution des normes qui la régit.

Il faut préciser toutefois que, à l'heure d'Internet et des réseaux sociaux, les open mics ne constituent qu'une porte d'entrée parmi d'autres dans le domaine du rap. Ce type de dispositif trouve en effet écho avant tout chez les artistes désireux de mettre en avant la 
prestation live et les interactions en face à face avec d'autres membres de la scène. Néanmoins, l'investissement d'un segment de la scène ou d'un autre ne se fait pas de manière exclusive : un certain nombre de rappeurs doublent ainsi leur fréquentation des open mics d'un investissement intense des réseaux sociaux ou des sites de partage de vidéos. Les organisateurs des événements eux-mêmes tendent de plus en plus à effectuer un enregistrement vidéo de certains moments forts de leurs soirées qu'ils partagent par la suite sur les réseaux.

\section{L'approche par les pratiques spatiales pour aborder la scène}

Les pratiques spatiales présentes dans les open mics constituent ainsi un bon point de départ pour observer les scènes locales du rap. Par leur inscription dans un espace physique et social donné, les pratiques sont un révélateur de la scène et des normes qui la régissent. Mais elles contribuent également à forger les représentations associées au lieu, participant en cela à la construction de la scène en tant qu'espace symbolique. Elles agissent alors en tant qu' « 'agent' performatif [...] dans la construction de territoires et de sense of place » (Guiu, 2006).

Ces pratiques étant abordées ici dans le cadre d'un événement de type concert, elles seront observées sous deux angles différents.

On abordera d'une part les «performances » qui se déroulent dans les open mics. Ce terme, largement utilisé depuis quelques temps en géographie, désigne « la pratique en situation d'un individu, en ce qu'elle incarne des normes socio-culturelles qui la régissent mais aussi en ce qu'elle participe elle-même à la reproduction et/ou à la subversion de ces normes » (Chapuis, 2012, p. 26). Il faut préciser que le terme de performance ne sera pas utilisé ici pour se référer uniquement au spectacle se déroulant dans l'espace scénique. II servira en effet à qualifier plus généralement l'ensemble des interactions entre acteurs ayant lieu durant l'événement, en considérant, à l'instar de Goffman, que même les pratiques les plus anodines peuvent participer d'un processus de mise en scène de soi (1973a et 1973b).

19 D'autre part, seront analysés les discours délivrés par les acteurs sur leur pratique. Il s'agit en effet de ne pas se limiter à une interprétation des performances donnée par le chercheur, mais d'observer également le sens qu'y attribuent les personnes fréquentant ce type d'événement. Cet aspect permettra notamment de s'attacher à la dimension symbolique des open mics, en analysant la fonction qu'ils possèdent au sein de la scène, et l'influence qu'ils ont sur celle-ci.

\section{Le cadre de l'enquête : économie et format des open mics dans les capitales du rap}

20 Les résultats présentés dans cet article se fondent sur des enquêtes de terrain menées entre août 2012 et janvier 2014 à Paris et à Atlanta. Ces deux villes ont été choisies pour leur statut de capitale pour ce genre musical. Comme dans beaucoup d'autres secteurs artistiques, la région parisienne constitue en effet un « centre de gravité » (Menger, 1993) du rap français, étant donné la concentration importante de labels, de médias et de salles de concert que l'on y retrouve. La région d'Atlanta correspond également à un «carrefour " pour l'industrie du rap aux Etats-Unis. Elle a par ailleurs occupé un rôle 
particulièrement important dans la montée en puissance d'un rap du Sud et de l'esthétique qui lui est associée (Miller, 2010).

Les enquêtes sur place ont consisté en des observations dans plusieurs événements à Paris et à Atlanta, des entretiens (formels ou informels) avec des organisateurs et des artistes, et de l'analyse de matériau documentaire lié à ces soirées.

Ce travail a été effectué dans le cadre plus général d'une thèse en géographie, portant sur l'inscription dans la ville du rap français et américain, qui a donné lieu notamment à la réalisation de plus d'une trentaine d'entretiens dans les deux villes. Si ce travail ne concernait pas spécifiquement les open mics au départ, la thématique est néanmoins apparue progressivement, au fil des entretiens, jusqu'à constituer un cas d'étude spécifique.

3 Concernant les open mics observés, trois séjours à Atlanta, d'un à trois mois chacun, ont été l'occasion d'une fréquentation régulière de ce type de soirées. Trois événements ${ }^{5}$ en particulier ont été suivis à plusieurs reprises et ont constitué la base de ce travail. Dans cette ville, l'absence d'entretiens formels auprès des organisateurs a été compensée par une étude systématique de leur discours sur les réseaux sociaux et durant la tenue des événements. Par ailleurs, la question des open mics a également donné lieu à de multiples discussions avec des artistes participants ou leurs manageurs, lors d'entretiens enregistrés ou de moments plus informels, sur la signification de tels événements.

4 A Paris, deux open mics ont été observés sur la longue durée. Ces éléments ont été complétés par une fréquentation ponctuelle d'autres événements du même type, ainsi qu'un travail documentaire sur le sujet afin d'évaluer la représentativité des éléments observés. Un entretien a été fait spécifiquement avec les organisateurs de chaque événement suivi sur la durée. Si, de même qu'à Atlanta, aucun entretien avec des rappeurs ne s'est focalisé uniquement sur les open mics, cette thématique a cependant été soulevée de manière approfondie dans plusieurs des entretiens formels et informels effectués auprès d'artistes dans le cadre plus général du travail de thèse.

Le format de l'ensemble des open mics identifiés dans chacune des villes s'est avéré très divers dans les deux scènes, même si l'on peut observer certaines tendances dans chaque terrain d'étude. Ainsi, à Atlanta, les événements semblent s'inspirer la plupart du temps du modèle des showcases : les artistes arrivent avec un produit fini (un ou plusieurs morceaux), qu'ils doivent interpréter ou passer tel quel sur scène après s'être brièvement présentés. A Paris, les open mics témoignent d'une moins grande importance accordée au produit fini. Entre tremplin et battle, ils sont plus orientés autour de la compétition entre rappeurs et laissent souvent plus de place aux formes d'improvisation rappées, les freestyles. Ainsi, lorsque les artistes rappent sur un instrumental, celui-ci peut parfois être choisi par le DJ. La compétition entre rappeurs, en revanche, ne prend pas forcément la forme d'une confrontation face à face mais plutôt celle d'une évaluation par le public ou un jury extérieur.

6 Il existe également certaines différences entre les concerts des deux villes en terme économique. Si, à Paris, il est généralement demandé aux artistes désirant se produire de s'acquitter uniquement du droit d'entrée et de s'inscrire sur une liste, à Atlanta certains événements demandent le paiement d'un droit d'inscription pouvant monter jusqu'à une centaine de dollars pour présenter seulement quelques morceaux.

Dans les deux cas, cependant, les open mics regroupent majoritairement un public fidèle et averti. En effet, certains processus de diffusion de l'information font qu'il est parfois 
difficile d'assister à un open mic si l'on n'est pas déjà investi au préalable dans le monde du rap $^{6}$. Par ailleurs, la forme de ces événements fait qu'ils sont particulièrement utilisés pour faire ses premières armes dans le monde du rap. Ils constituent ainsi des espaces où va s'opérer une construction de la légitimité, selon des procédés qui révèlent certaines des logiques selon lesquelles s'articulent les différentes scènes.

\title{
«Started from the bottom now we here ${ }^{7}$ »: les pratiques des open mics à Atlanta entre performance et performativité
}

\author{
«Yeah yo, imaginons qu'on est en '98 \\ Que je mange mon repas dans des plateaux en mousse \\ Que j'essaye d'être le prochain rappeur à sortir d'Atlanta (A-Town) \\ Espérant une signature sur un label pour étancher ma peine » \\ «Yeah yo, let's pretend like it's '98 \\ Like I'm eatin' lunch off of Styrofoam trays \\ Trying to be the next rapper coming out the A (A-Town) \\ Hoping for a record deal to ignore my pain " \\ (B.o.B.,« Airplanes Part. 2.», The Adventures of Bobby Ray, Rebel Rock/Grand \\ Hustle/Atlantic)
}

A Atlanta, les open mics sont le lieu de performances assez singulières qui, de prime abord, semblent envoyer des signaux contradictoires. Ainsi, à l'instar ce qu'à pu effectuer Djemila Zeneidi-Henri dans son analyse sur les relations de genre dans le monde du punk (2005), il est nécessaire de procéder à plusieurs niveaux de lecture afin de saisir l'entière complexité de ce qui s'y joue.

\section{Des lieux de mise en scène de la célébrité...}

Pour l'observateur extérieur, les open mics à Atlanta présentent à première vue les signes d'événements situés au centre d'un star system local.

Ils sont caractérisés tout d'abord par la présence d'un éventail relativement large des personnels investis dans le monde du rap. Parmi eux, celle de journalistes semble témoigner de l'importance que l'événement a l'air de revêtir : ils constituent en effet des acteurs particulièrement visibles au sein des open mics, de par les flashs imposants des appareils photo qu'ils arborent, ou les interviews qu'ils effectuent en marge du concert. Or, à l'inverse des pratiques habituelles présentes dans les concerts, leurs appareils ne sont pas orientés vers l'espace scénique, mais vers l'audience. En plus de donner l'impression que l'open mic accueille des personnalités particulièrement importantes dans la scène rap locale, cet élément amène à penser qu'il ne s'agit pas forcément de celles en train de rapper devant le public.

On constate par ailleurs dans les open mics une mise en scène vestimentaire et corporelle individualisée et marquée. Celle-ci ne semble pourtant pas révéler l'appartenance à un collectif, comme dans le cas des punks (Zeneidi-Henri, 2005). Leur variété fait en effet plutôt penser à un désir d'être remarqué individuellement, dans une logique assez proche des défilés lors des remises de prix dans l'industrie musicale ou cinématographique. Des codes correspondant à ce monde sont d'ailleurs repris dans certains concerts à Atlanta, comme la présence de séances photo devant un panneau publicitaire mentionnant les différents sponsors de la soirée. 
Ces impressions sont confirmées par le discours des présentateurs. Ceux-ci annoncent souvent les artistes passant sur scène avec une emphase particulière, en précisant à quel point il est exceptionnel de les voir maintenant, étant donné le succès dont ils bénéficieront rapidement. Les rappeurs eux-mêmes, avant de se produire, prennent généralement soin de détailler leur impressionnant $\mathrm{CV}$, mentionnant le nombre de vues de leurs clips sur Youtube, ou les collaborations qu'ils ont pu faire avec des têtes de file de la scène d'Atlanta. Tout se passe donc comme si les open mics accueillaient le défilé d'un ensemble de stars, ou a minima, de personnes déjà engagées sur la ligne droite du succès commercial.

\section{... par des individus en quête de reconnaissance}

Pourtant, d'autres aspects des performances contrastent avec la description effectuée précédemment.

Ces éléments se retrouvent tout d'abord dans les comportements des journalistes. Après avoir pris une photo, ceux-ci abordent généralement leur sujet en leur confiant leur carte de visite. En effet, au même titre que les rappeurs cherchant à faire connaitre leurs produits musicaux, les journalistes présents sont généralement des bloggeurs débutants en recherche de collaborations.

On peut retrouver également au sein des open mics d'Atlanta des éléments qui ne sont habituellement pas associés à l'espace de la salle de concert. Ils rappellent en cela l'analyse effectuée par Goffman à propos des espaces de la performance. Celui-ci distingue deux "régions " où s'opèrent des mises en scène de soi bien distinctes : les régions antérieures (front regions), dans lesquelles un individu se place en représentation par rapport à d'autres personnes, et les régions postérieures (back regions), correspondant aux coulisses où l'individu peut se laisser aller à d'autres types de comportements, ou préparer la représentation en devenir (Goffman, 1973a). Or, cette distinction semble quelque peu trouble dans le cadre des open mics. Ainsi, dans un des concerts observés, on pouvait trouver autour des lavabos des toilettes un ensemble de produits de maquillage destinés aux artistes, choses habituellement présentes dans des loges non accessibles au public. En plus de confirmer l'importance accordée à la mise en scène de soi chez les personnes présentes, cet élément montre également que celle-ci correspond à un code explicitement affirmé par les organisateurs.

\section{La mise en scène de soi comme expression performative}

Les signaux contradictoires véhiculés par les performances incitent ainsi à procéder à un troisième niveau de lecture. Les pratiques doivent pour cela être mises en perspective avec le but recherché par les personnes qui les mettent en place. L'explication n'est alors pas tant à chercher du côté de la performance que de celui de la performativité. Les mises en scène des individus présentes dans les open mics à Atlanta constituent en effet un bon exemple de "pratiques réitérées et citationnelles par lesquelles les discours produisent les effets qu'ils décrivent ${ }^{8} \gg$ (Butler, 1993).

Pour cela, il faut d'abord observer les liens de ces événements avec le monde de l'industrie musicale. Deux des principaux open mics observés, chacun d'une périodicité mensuelle, étaient en effet organisés par l'un des principaux labels de la ville. Mais c'est 
surtout la présence de membres éminents de l'industrie musicale durant l'événement qui est mise en avant durant les soirées.

A cet égard, il est intéressant d'observer le type de discours publiés sur Facebook par les propriétaires de la page consacrée à l'un des événements observés, le Spotlight Open Mic ${ }^{9}$, organisés par l'un des principaux labels de rap d'Atlanta (cf. encadré). Il y est annoncé que l'événement suscite l'intérêt des personnes travaillant dans l'industrie musicale, qui assistent aux concerts à la recherche de personnes à signer. Celles-ci ne sont d'ailleurs pas les petites mains de ces labels mais bien celles qui prennent des décisions (les " executives») et façonnent les goûts musicaux de demain (les "tastemakers»). Dans ce contexte, se produire à cet événement est présenté comme une opportunité considérable pour devenir la prochaine star du rap américain.

Ces discours sont repris durant les soirées par les présentateurs. Il y est régulièrement mentionné que plusieurs personnalités importantes se trouvent dans la salle. Parfois mentionnées nommément, elles sont le plus souvent présentées comme dissimulées dans le public, ne se dévoilant que si elles venaient à détecter un «vrai talent». Il en résulte ainsi un encouragement d'une mise en scène de soi permanente, les organisateurs rappelant régulièrement que l'« on ne sait jamais qui vous regarde» («you never know who is watching you »).

Quelques exemples de statuts publiés sur la page Facebook du Spotlight Open Mic (Atlanta, oct.-nov. 2013)

«You never know what industry tastemakers in the building TONIGHT! Come out \#NETWORK » (10/10/13)

« The opportunities are real.. [Spotlight]OpenMic can be a life changing experience!! $11 / 14 »(29 / 10 / 13)$

« November 14th a superstar will be found! Is that you?» $(01 / 11 / 13)$

«****** from Atlantic records was in the building last month... There is no telling who will be there on the $14^{\text {th }}$ » $(01 / 11 / 13)$

«Execs from Sony, Interscope, Atlantic and Def Jam have all been to @ [Spotlight]OpenMic, scouting for talent! Be there 11/14»(02/11/13)

«We rarely put our invited execs on the spot, once they hear something they like they always get with the artist @[Spotlight]openMic 11/14» (publié le 04/11/13)

C'est dans ce contexte que l'on peut mieux comprendre les performances mises en place par des artistes. Au même titre que pour le rock à Liverpool (Cohen, 1991), le rap à Atlanta représente une opportunité économique aussi acceptable que de chercher un emploi à plein temps dans d'autres secteurs d'activités. Le coût d'entrée dans le domaine du rap est en effet peu élevé et le contexte économique est présenté comme particulièrement favorable, étant donné les représentants de labels qui parcourent la ville. Dans ce cadre, les open mics constituent des occasions particulièrement intéressantes : en plus de constituer des infrastructures efficaces pour se produire à moindre coût, ils permettent également d'entrer en contact avec des membres de l'industrie musicale, avec l'espoir d'obtenir une signature sur un label.

Cependant, si les opportunités économiques à la clé sont extrêmement conséquentes, seul un petit nombre de personnes est sélectionné parmi l'ensemble des prétendants. Il en 
résulte donc une concurrence extrêmement importante au sein même de la scène pour arriver en haut de l'échelle.

Dans ce contexte, les performances des artistes constituent une manière de se distinguer des autres candidats potentiels. L'objectif est en effet de se faire remarquer par les quelques personnes qui comptent dans l'assemblée en montrant, par une certaine mise en scène de soi, que l'on a la carrure pour être la prochaine star. Pour cela, le moyen le plus couramment utilisé est de montrer, par différents stratagèmes, que l'on a déjà réussi sa carrière avant même de la commencer.

Cette logique est résumée au sein de la scène par l'expression " fake it 'till you make it " ${ }^{10}$. L'idée est que, si l'on arrive suffisamment bien à incarner une certaine identité correspondant au statut que l'on désire acquérir, il serait possible que certaines personnes finissent par se prendre au jeu, et que l'apparence de succès que l'on donne à voir se traduise ensuite par des opportunités économiques réelles.

Ce contexte va ainsi pousser les performances dans deux directions. D'un côté, les rappeurs vont chercher à se conformer à ce qu'ils imaginent être les exigences de l'industrie musicale qu'ils cherchent à toucher. De l'autre, ils vont tenter de montrer en quoi ils se distinguent d'une foule d'autres candidats potentiels, et pour quelle raison ils possèdent plus de légitimité à être remarqué. Dans les deux cas, ces stratégies témoignent cependant d'une anticipation d'attentes venant du haut.

\section{« Faire partie des vrais »: les open mics à Paris et la rhétorique de la légitimité}

"On en a fait du chemin, rappelle-toi

En 2009 brûler les open mics entouré par 5-6 vrais gars"

(Fonky Flav, du groupe 1995, "Comme un grand », La Suite, Polydor)

À première vue, les open mics à Paris présentent de nombreux points communs avec ceux d'Atlanta. Dans les entretiens, ce type d'événements est ainsi présenté comme des « détecteur[s] de talent », permettant aux artistes de «se faire connaitre » ou d'acquérir un «buzz».

Pourtant, si le fonctionnement général est le même, il existe certaines différences dans les performances qui s'y déroulent. La plupart des événements se fondent en effet avant tout sur des improvisations des rappeurs, ne permettant pas forcément le même type de mise en scène. Les performances mises en place ne sont donc pas forcément celles qui sont à même de séduire un label à la recherche d'un produit fini. Dans l'un des entretiens effectués avec un organisateur, celui précisait ainsi que, si les personnes intervenant dans ses open mics sont pour la plupart des "artistes en développement", ceux-ci ont cependant « autre chose à leur carte qu'un bon freestyleur sur scène » (Entretien réalisé le 22/01/13).

Le contexte dans lequel se déroulent ces événements présente également une autre différence notable par rapport à Atlanta. En effet, la majorité des open mics auxquels j'ai $\mathrm{pu}$ assister étaient organisés par des personnes certes investies dans le milieu du rap, mais à des postes extérieurs à l'industrie musicale. S'il est de notoriété publique que quelques individus travaillant dans ce secteur fréquentent de temps à autre les open mics, les organisateurs déclarent pourtant ne leur accorder qu'une attention marginale, voire être même incapables de les reconnaitre. 

que ceux-ci revêtent au sein de la scène parisienne. Or, à Paris, la signification accordée à ces événements se retrouve de manière particulièrement prégnante dans les discours qui accompagnent les pratiques.

\section{Pratiques spatiales et vocable symbolique}

Paris, la fréquentation des open mics se double de tout un vocable symbolique. Un des organisateurs interrogés donne ainsi l'explication suivante à la fréquentation intensive de ces événements par certains artistes :

"C'est pas compliqué. Toutes les scènes plus ou moins accessibles, ouvertes à tous, on retrouve toujours les mêmes. Parce que c'est les mecs qui ont la dalle, et c'est ça la Street Crédibilité. » (Entretien réalisé le 22/01/13)

Dans cet extrait se retrouvent deux niveaux de discours. L'emploi de l'expression « avoir la dalle" suggère ici que les open mics sont avant tout investis par des personnes dépourvues d'opportunités économiques, et donc a priori marginales dans le monde du rap. Cependant, l'association des pratiques de fréquentation intensive à la «street crédibilité » suggère un sens bien différent. Cette expression s'intègre en effet dans le cadre d'un ensemble de références à la «rue» utilisé pour définir «les formes acceptables de coopération" (Hammou, 2009) associées au genre rap dans la région parisienne. La rue désigne au départ, dans le discours des acteurs du rap ou même chez les chercheurs en sciences sociales, le lieu d'origine, réel ou mythique, des pratiques hiphop. Dans cette acception, le terme correspond à un espace physique et symbolique, celui de la socialisation d'une jeunesse masculine précarisée, mais aussi de certaines pratiques délinquantes. Cependant, selon Karim Hammou, son usage fait l'objet d'un glissement symbolique dans le monde social du rap français à partir des années 2000. A cette période, on assiste en effet à une professionnalisation de ce genre musical, qui se traduit par l'émergence de lieux définissant le rap comme un genre qui leur est propre. Ces lieux s'établissent en opposition aux espaces dominants de l'industrie musicale, dans lesquels le rap reste maintenu au rang de pratique minoritaire. Les lieux qui définissent le rap comme un genre qui leur est propre ont ainsi pour objectif d'être des endroits où, contrairement à d'autres, les normes du rap peuvent s'exprimer pleinement.

Dans ce cadre, les références à la rue, et aux valeurs qui y sont associées, sont transposées au domaine musical pour qualifier les pratiques se déroulant dans ces lieux du rap. Ce glissement résulte d'une analogie effectuée entre le domaine musical et le reste de la société : de la même manière que la rue désigne un espace accueillant des populations marginalisées dans la société, les lieux du rap accueillent un genre possédant un statut minoritaire au sein des circuits dominants de l'industrie musicale.

De plus, dans la mesure où la rue désigne l'espace de référence pour la pratique du rap, son utilisation pour qualifier les pratiques au sein de lieux spécifiques permet de les ériger comme les endroits dans lesquels va se forger la crédibilité des rappeurs. En ce sens, le vocable de la rue constitue un "symbole honorifique» servant à qualifier l'« authenticité » des lieux et des personnes qui les pratiquent dans le monde social du rap (Hammou, 2009).

Or, ce discours se retrouve ici dans le cadre des open mics. L'emploi du terme de «street crédibilité » pour qualifier ce type d'événement permet en effet, dans une logique performative, de les placer symboliquement au centre de la scène. 
54 A Paris, le discours autour des open mics s'inscrit ainsi dans un processus de retournement des valeurs. L'enjeu réside en effet dans le fait de montrer que, bien qu'éloignés de la sphère de l'industrie musicale et de ses opportunités économiques, ces événements ne constituent pas une ressource par défaut, mais bien les hauts lieux de la scène.

On constate donc la mise en place de toute une rhétorique performative permettant d'affirmer la légitimité de ces événements : les rappeurs se produisant dans ce type d'événements sont qualifiés de "vrais » ou "d'authentiques" et les organisateurs ne cessent d'affirmer que c'est la fréquentation de leurs événements qui va permettre d'évaluer la crédibilité des rappeurs.

56 Ainsi, de simples tremplins, les open mics à Paris sont peu à peu devenus des lieux à investir au sein de la scène, notamment pour les anciens rappeurs désireux de se refaire un nom. Dans un article du Monde, un membre du groupe Expression Direkt, de retour après 8 ans d'absence, justifie ainsi sa volonté de venir jouer dans un open mic organisé à la Miroiterie en juin 2012 :

«Les scènes ouvertes comme les battles [joutes verbales], ce sont les fondations du hip-hop [...]. On vient pour voir ce que les connaisseurs pensent de nos nouveaux textes. » (Weedy, cité dans Binet, 2012)

\section{Les open mics comme arènes de la légitimité}

Les critères d'évaluation affichés sont cependant bien différents de ce que l'on pouvait observer à Atlanta. Alors que, dans cette dernière ville, il s'agissait de convaincre des personnes clés dans le domaine de l'industrie musicale, c'est ici le public qui va servir de juge aux prestations des rappeurs. Le terme "connaisseur ", employé dans la citation précédente par le rappeur d'Expression Direkt, s'insère ainsi dans toute une batterie de termes semblables utilisés pour le qualifier, tels ceux de "puristes ", de "public averti ", d'« aficionados ", voire même de "fanatiques ». Ces dénominations rappellent la catégorie de l'«amateur ", identifiée par Stéphanie Molinero dans ses travaux sur le public du rap français. Celle-ci a en effet montré que, parmi les auditeurs de rap, se retrouvait un type de personnes revendiquant un jugement esthétique sur cette musique et fréquentant assidument les événements qui lui sont consacrés (Molinero, 2009).

Or c'est justement ces "amateurs" que les rappeurs cherchent à atteindre en se produisant dans les open mics. Il s'agirait selon les rappeurs de la catégorie de personnes légitime pour évaluer la qualité d'une prestation car ils seraient situés au centre du monde social du rap français.

Dans ce cadre, les open mics sont présentés comme des événements permettant d'avoir l'aval d'un réseau de pairs, comme une arène dans lequel le rappeur va pouvoir forger sa crédibilité. Par un phénomène de métonymie, être reconnu au sein des open mics reviendrait avoir l'aval de l'ensemble du monde du rap français. Ainsi, l'un des organisateurs décrit son événement de la manière suivante :

«Si en France, tu veux prouver que t'es un bon, si tu vas là et que tu es fort, et ben tu peux rapper n'importe où en France et en Europe. Je le dis sans pitié. Si tu déchires là, c'est le public le plus compliqué. Mais c'est le plus écouteur et le plus souteneur. » (Entretien réalisé le 21/01/14)

Or, cette situation a des implications sur les types de performances présentes au sein des open mics. On pourrait pourtant penser que, contrairement à Atlanta où l'industrie musicale impose une identité dominante contraignante pour les artistes, les open mics 
parisiens sont plus ouverts à différents types de tendances. C'est d'ailleurs la thèse défendue par certains chercheurs, Stéphanie Molinero affirmant par exemple que la sphère underground du rap français constitue un « laboratoire artistique » où se font les propositions artistiques novatrices (2009). Cette thèse se retrouve également chez les organisateurs d'open mics, qui déclarent généralement que le seul critère pour réussir dans un open mic est d'être "bon ». Ce jugement esthétique s'aligne cependant sur des mécanismes de distinction au sein de la scène reproduisant des rapports de domination. Ainsi, l'un organisateur interrogé déclare à propos de son événement :

\begin{abstract}
«C'est ouvert pour tout le monde. Même si t'as enregistré ou pas enregistré [d'album], on s'en fout. Si tu es amateur complet et même nul, peu importe. Si tu es inscrit dans les temps, tu passes et tu auras l'occasion de te confronter à la dure réalité de monter sur scène pour être mauvais. Tu verras ce que ça veut dire. Et tu apprendras et, quand tu reviendras, tu feras pas pareil. Après, il y en a qui se pointent, tu sens très clairement que c'est du rap ghetto, vénère, et ils passent pas l' open mic. Et c'est à eux de comprendre que rapper pour soi et représenter son quartier, ça a ses limites, qui sont que tu es pour un public qui n'est pas acquis à ta cause, et que c'est à toi de trouver le chemin pour les mettre dans ta poche.» (Entretien réalisé le 22/01/13)
\end{abstract}

61 Ainsi, l'aval du public semble s'aligner là encore sur un certain nombre de normes, qui implique une sélection d'un certain profil d'artistes plutôt qu'un autre. En effet, ce discours de l'organisateur peut être mis en parallèle avec une volonté de sa part d'organiser son événement à Paris intra-muros, certes pour des questions d'accessibilité et pour l'aspect symbolique, mais également pour que l'événement ne soit pas trop " difficile à gérer ».

\title{
Les open mics comme révélateurs de deux modèles de capitales du rap
}

62 A Paris comme à Atlanta, les open mics étudiés mettent ainsi en évidence deux modèles de capitales du rap, qui reflètent la manière dont se construisent et structurent des espaces de la production artistique dans plusieurs domaines.

\section{La construction de la légitimité musicale}

63 Les open mics apparaissent comme des espaces où se construit la légitimité artistique, dans deux scènes où sa définition et son origine ne sont pas les mêmes. Cela se retrouve ainsi dans les discours performatifs qui transparaissent dans les performances du corps et les paroles des artistes à Atlanta et à Paris : tandis que, dans la première ville, l'objectif est d'atteindre la signature sur un label, dans l'autre, il s'agit de défendre sa crédibilité en fréquentant l'archipel des lieux définissant le rap comme un genre qui leur est propre (Hammou, 2009). D'un côté, il s'agit ainsi d'anticiper des normes venant d'en haut, tandis que, de l'autre, il est question de s'intégrer à un réseau de pairs.

64 Mais la question de la légitimité se pose également pour la place des événements en euxmêmes au sein de la scène. Alors qu'à Atlanta, ils semblent être périphériques dans une scène où le centre correspond au monde de l'industrie musicale, on constate à Paris une rhétorique de la légitimité qui vise à les replacer au centre, en montrant qu'il s'agit des lieux dans lequel s'établissent les valeurs du genre rap français. 


\section{L'ancrage de l'industrie musicale}

65 « latino", Atlanta est associée dans l'industrie musicale aux «musiques urbaines». Les A\&Rs présents sont spécialisés en priorité dans ce type de musique et les structures locales sont avant tout centrées autour de ce secteur musical. En d'autres mots, l'industrie musicale à Atlanta correspond peu ou prou à l'industrie du rap. A Paris, la situation est bien différente. Cette région étant une capitale artistique dans la majorité des domaines artistiques et musicaux, le rap n'y est qu'un genre musical parmi d'autres. C'est dans ce contexte que l'on peut comprendre la centralité d'un monde social indépendant au sein de la scène.

69 Il faut noter cependant que la situation étudiée correspond cependant à des moments spécifiques des scènes. La scène rap d'Atlanta s'est en effet trouvée dans une conjoncture particulièrement favorable dans le monde du rap américain ces dernières années. En effet, entre 2007 et 2012, sur un total de 64 albums de rap ayant atteint le disque d'or, 10 étaient le fait d'artistes provenant d'Atlanta, la plaçant au $1^{\text {er }}$ rang, devant New York (9 albums) et Los Angeles (7 albums) ${ }^{12}$. Dans ce cadre, on peut se demander si Atlanta ne correspond pas à une «bulle spéculative » sur laquelle misent les maisons de disque, qui plus est dans un pays où les grandes maisons de disques procèdent à des stratégies de recherches localisées. 
70 Si ce type de configuration n'était pas présent dans le cas parisien au moment du terrain, sa situation serait cependant à replacer également dans une perspective temporelle. Bien que la structuration géographique de l'industrie musicale française ne permette pas l'orientation majoritaire de la région parisienne autour d'un genre musical spécifique, rien n'empêche que la scène rap puisse constituer à certaines périodes une bulle au sein du paysage musical francilien sur laquelle les maisons de disque peuvent être amenées à parier. Dans ce cadre, l'établissement de liens directs entre les organisateurs des événements et des grandes maisons de disque, des médias généralistes ou des programmateurs musicaux pourrait, à l'instar d'Atlanta, conduire les open mics à se changer pour un temps en tremplins pour les artistes dans le monde de l'industrie musicale.

\section{Les régimes d'authenticité du rap}

71 L'étude des open mics permet ainsi de réexaminer sous un autre jour l'élaboration des codes propres à ce genre musical.

72 En effet, à l'instar d'autres genres musicaux, la correspondance des artistes aux conventions du genre rap est régulièrement évaluée sous le registre de l'authenticité (Forman, 2002). Or, il a largement été montré dans les recherches sur les musiques populaires comment cette authenticité ne résultait non pas de caractéristiques propres à l'artiste, mais de sa validation comme telle par un groupe, selon des critères esthétiques historiquement et culturellement situés. En ce sens, la construction de l'authenticité correspondrait donc avant tout à un processus d'authentification (Moore, 2002).

L'analyse des open mics de rap permet d'aller plus loin dans ce domaine, en montrant que cette construction d'authenticité résulte aussi de facteurs locaux. Ainsi, plutôt que de considérer qu'il existerait pour l'ensemble du genre rap des codes préétablis auxquels les artistes devraient se conformer pour paraitre « authentiques », on peut avancer l'idée que la production de cette musique dépend au contraire de "régimes d'authenticité " élaborés au sein de chaque contexte local.

74 L'idée de « régime " permet en effet de mettre l'accent sur le fait que la production de l'authenticité, ici à l'échelle locale, résulte d'une construction collective et non d'une simple production individuelle. En cela, elle met en évidence ce que "l'identité locale » du rap doit aux interactions entre acteurs se déroulant au sein d'un monde artistique localisé.

75 Elle sous-tend par ailleurs l'existence d'un ensemble de mécanismes, de pratiques et de procédés structurant des routines et des rythmes de fonctionnement. Bien que l'analyse puisse donner l'impression d'un ensemble relativement stable, ces «régimes d'authenticité » doivent être compris comme une structure en perpétuelle évolution, en fonction de l'histoire de la scène locale, de la représentation dominante qui en existe au moment où un artiste s'y insère et de la place qu'elle occupe à l'échelle nationale.

Ainsi, l'étude des open mics au sein des scènes rap de Paris et à Atlanta met en valeur la structuration d'un espace de la production artistique, ses normes, ses centres et ses périphéries. Mais elle met également l'accent sur la manière dont se construisent les représentations qui vont se retrouver dans les produits musicaux, s'exporter à l'extérieur de la scène, et influencer en retour les pratiques à l'échelle locale. 
Les résultats de cette étude donnent également des éléments sur la fonction de capitale occupée par Paris et Atlanta dans le monde du rap. Faire porter l'analyse sur ces deux villes en les considérant comme des "centres » du rap permet ainsi de montrer ce que cette musique, y compris dans ses principaux foyers, doit à des facteurs locaux. Dès lors, si les résultats présentés apportent des informations sur deux localités, ils ne sauraient être considérés comme représentatifs des différences existantes entre le rap " français » et le rap "américain» dans leur ensemble. L'exceptionnalité de ces deux villes est notamment fondée sur leur statut de capitale du rap. Dans ce contexte, l'étude présentée ici invite à reconsidérer les espaces nationaux de la production du rap sous l'angle des relations entre centres et périphéries, en complétant les études sur les capitales du rap par celles de pôles plus secondaires. Ce n'est qu'ainsi que l'on pourra cerner avec plus de complexité l'entière spécificité des espaces de production du rap qui constituent des mondes artistiques à part entière.

\section{BIBLIOGRAPHIE}

BENNETT A., PETERSON R. (2004), Music Scenes. Local, Translocal and Virtual, Nashville, Vanderbilt University Press.

BENNETT A. (2004), « Consolidating the music scenes perspective «, Poetics, 32, Amsterdam, Elsevier, pp. 223-234.

BINET S. (2012), « Rap = MCs : les 'maîtres de cérémonie' à Paris et leur 'battles' sans merci« , Le Monde, 26/06/12, p. 22.

BUTLER J. (1993), Bodies that matter: On the discursive limits of « sex », New York, Routledge.

CHAPUIS A. (2012), Performance touristiques et production des identités spatiales individuelles à Amsterdam, Thèse de doctorat, Université Paris 1.

COHEN S. (1991), Rock Culture in Liverpool: Popular Music in the Making, Oxford, Clarendon Press.

FORMAN M. (2002), The hood comes first: race, space and place in rap and hip-hop, Middletown, Wesleyan University Press.

GOFFMAN E. (1973a), La mise en scène de la vie quotidienne. T.1 : La présentation de soi, Paris, Editions de minuit.

GOFFMAN E. (1973b), La mise en scène de la vie quotidienne. T.2 : Les relations en public, Paris, Editions de minuit.

GUIU C. (dir.) (2006), « Géographie et Musique, quelles perspectives ? , Géographie et Cultures, 59, Paris, L'Harmattan.

HAMMOU K. (2009), Batailler pour un chant. La constitution d'une culture professionnelle musicienne du rap français, Thèse de doctorat, EHESS.

HAMMOU K. (2014), « Le deuxième marché mondial... Vraiment ? , Sur un son rap, en ligne sur http://surunsonrap.hypotheses.org/3038, publié le 14/07/14. 
MENGER P.-M. (1993), «L'Hégémonie parisienne. Economie et politique de la gravitation artistique », Annales. Économies, Sociétés, Civilisations, 6, pp. 1565-1600.

MILLER M. (2010), «'The Sound of Money': Atlanta, crossroad of the Dirty South », in HESS M. (ed), Hip-Hop in America : A Regional Guide, Volume 2: The Midwest, the South, and Beyond, Santa Barbara, Denver, Oxford, Greenwood Press, pp. 467-494.

MITCHELL T. (ed.) (2001), Global Noise. Rap and Hip-Hop Outside the USA, Middletown, Wesleyan University Press.

MOLINERO S. (2009), Les Publics du rap, enquête sociologique, Paris, L'Harmattan.

MOORE A. (2002), « Authenticity as authentification », Popular Music, 21, 2, Cambridge, Cambridge University Press, pp. 209-223.

RAIBAUD Y. (2011), « Musique noire : la musique des Afriques dans le monde », in RAIBAUD Y. (dir.), « Géographie des musiques noires », Géographie et cultures, 76, Paris, L’Harmattan, pp. 3-11.

RÉRAT P. (2006), « Le rap des steppes : l'articulation entre logiques globales et particularités locales dans le hip-hop mongol ", Géographies et Cultures, 59, Paris, L’Harmattan, pp. 43-56.

ROSE T. (1994), Black noise. Rap music and black culture in contemporary America, Middletown, Wesleyan University Press.

STRAW W. (1991), « System of Articulation and Logic of Change: Communities and Scenes in Popular Music », in Cultural Studies, 5, 3, London, New York, Routledge, pp. 368-388.

ZENEIDI-HENRY D. (2005), « Les punks ou la comédie des genres : une analyse à l'épreuve des pratiques spatiales et corporelles », Géographie et Cultures, 54, Paris, L'Harmattan, pp. 85-102.

\section{NOTES}

1. "Cultural space in which a range of musical practices coexist."

2. "Clusters of producers, musicians, and fans collectively share their common musical tastes and collectively distinguish themselves from others".

3. "The making and remaking of alliances."

4. Ce terme est utilisé dans le milieu du rap pour désigner les artistes ou les groupes qui n'ont pas (encore) de succès commercial et opèrent donc hors des circuits de production et de distribution dominants de l'industrie musicale.

5. Les événements ne sont volontairement pas cités nommément afin de préserver l'anonymat des lieux et, par là même, des personnes concernées.

6. A Paris, l'organisateur de l'un des événements suivis assurait ainsi faire sa promotion uniquement par les réseaux sociaux et par mail, en fonction des contacts dont il disposait. A Atlanta, l'absence, au moment du terrain, d'un magazine papier ou d'un site internet annonçant l'ensemble des événements liés au rap contribue à un manque de visibilité de l'ensemble de l'offre d'open mics existant pour le public extérieur.

7. Drake, 2013, "Started from the bottom", Nothing was the same, Young Money, Cash Money Republic.

8. "Reiterative and citational practice by which discourse produces the effects that it names".

9. Le nom de l'événement a été modifié afin de préserver l'anonymat des événements observés et de leurs acteurs.

10. On pourrait traduire cette expression par «tricher jusqu'à ce que l'on réussisse » ou encore «fais semblant jusqu'à ce que ça se réalise ». 
11. Au début des années 2000, le marché du disque aux Etats-Unis générait ainsi, tous genres confondus, un chiffre d'affaires 15 à 25 fois plus important que dans le cas français (Hammou, 2014).

12. Source : https://www.riaa.com/

\section{RÉSUMÉS}

Le rap constitue un fait artistique dont la dimension spatiale a suscité assez tôt l'intérêt des chercheurs. Cependant, beaucoup d'études se sont contentées d'analyser les textes de rap, considérant qu'ils reflétaient de manière « naturelle » les modes de vie et les identités locales. Or, le rap correspond aussi à des mondes artistiques localisés dans lesquels vont s'élaborer les représentations reprises ensuite dans la musique. Cet article se propose ainsi de déplacer la réflexion vers les espaces de production du rap, en observant les pratiques spatiales dans des concerts de type open mic dans les régions urbaines de Paris et d'Atlanta. L'étude des performances et les discours qui les entourent permettront de mettre en évidence la construction de modes de légitimation différenciés au sein de deux scènes locales. On montrera ainsi que les open mics révèlent deux modèles de capitales artistiques, qui renseignent sur la structure et la hiérarchie des mondes du rap en France et aux Etats-Unis.

The spatial aspects of rap music have given birth to a whole body of literature in social sciences. However, a lot of studies approached this music through the songs, considering that they were "naturally" reflecting local ways of life and identities. Yet, rap music can also be regarded through local art worlds, where are created the representations that are then conveyed in the music. Thus, this paper will put the focus on the spaces of production of rap music, observing the spatial practices that take place in open mic shows in the urban regions of Paris and Atlanta. Through the study of performances and discourses displayed in these events it will be shown how the logics of legitimacy of rap music are built within two local scenes. In this way, open mics will highlight two models of artistic capitals that reveal a lot of the structure and the hierarchy of the rap music worlds in France and in the United States.

\section{INDEX}

Keywords : rap music, spatial practices, performance, legitimacy, scene

Mots-clés : rap, musique, pratiques spatiales, performances, légitimité, scène

\section{AUTEUR}

\section{SÉVERIN GUILLARD}

Université Paris Est, Lab’Urba, ATER, Université Paris 1, IREST, severin.guillard@gmail.com 